\title{
Intrathecal Morphine for caesarean delivery
}

\author{
Benedict Wallrich, MD¹, Katja Welsch², Thomas Volk, MD', Sven O. Schneider, MD1 \\ 1 Department of Anaesthesiology, Critical Care Medicine and Pain Therapy, Saarland University Hospital, Germany \\ ${ }^{2}$ Centre of Palliative Care and Pediatric Pain, The Saarland University Hospital, Homburg, Germany
}

Background: Caesarean delivery is often associated with strong postoperative pain and still leads in up to $18 \%$ to chronic postoperative pain. Intrathecal opioids are commonly used for analgesia and the reduction of needed local anaesthetics. We examined, whether the addition of morphine to the standard sufentanil has an impact on postoperative analgesia after caesarean delivery.

Methods: Using a before-and-after design we changed our standard protocol for spinal anaesthesia which includes $10 \mathrm{mg}$ bupivacaine hyperbaric and $5 \mu \mathrm{g}$ sufentanil by adding $100 \mu \mathrm{g}$ morphine. 153 patients undergoing caesarean delivery were examined. (83 with additional morphine, 70 without)

The extent of pain during the first 24 hours after surgery was the primary outcome measure. Additionally, side effects (nausea, dizziness, obstipation, pruritus, back pain, headaches) and the use of analgesics were compared. Data collection was accomplished via pain-out-questionnaire. For statistical analysis, the Mann-Whitney-U-Test was used.

Results: Evidence from this systematic investigation suggests no beneficial effect of additional morphine concerning maximum pain intensity, (VAS 0-10: 7,0 vs 7,0, median) but increased side effects like nausea, dizziness and pruritus.

Women who received morphine and sufentanil reported significant less restrictions in activity in bed $(7,0$ vs 7,5$)$ and in sleeping $(3,0$ vs 5,0$) \quad[0=$ no restrictions, $10=$ maximum restrictions]. They were more satisfied with the pain management $(8,0$ vs 7,0$)$ [0 very unhappy, $10=$ very pleased] and were given significant less paracetamol and tramadol than patients who only obtained sufentanil intrathecal.

Conclusion: Additional morphine has no benefit relating to maximum pain intensity whereas side effects are deteriorated. But women with additional morphine are more satisfied with pain therapy. These women seem to accept the side effects for less restrictions.

\begin{tabular}{|c|c|c|c|c|c|}
\hline & with morphine ${ }^{1}$ & Without morphine $^{1}$ & u-value & z-value & $p$-value ${ }^{2}$ \\
\hline maximum pain & $\begin{array}{l}7 \\
5,75-7,25 \\
\end{array}$ & $\begin{array}{l}7 \\
6,00-8,00 \\
\end{array}$ & 2296,50 & $-2,16$ & $\begin{array}{l}p=031 \\
p=.124\end{array}$ \\
\hline minimum pain & $\begin{array}{l}2 \\
1,00-3,00 \\
\end{array}$ & $\begin{array}{l}3 \\
1,00-3,00\end{array}$ & 2255,00 & $-1,80$ & $\begin{array}{l}p=.072 \\
p=.144\end{array}$ \\
\hline restrictions in activity in bed & $\begin{array}{l}7 \\
5,00-8,00\end{array}$ & $\begin{array}{l}7,50 \\
6,75-9,00\end{array}$ & 2132,50 & $-2,76$ & $\begin{array}{l}p=.006 \\
p=.030\end{array}$ \\
\hline $\begin{array}{l}\text { restrictions in taking a deep } \\
\text { breath or coughing }\end{array}$ & $\begin{array}{l}6 \\
3,00-8,00\end{array}$ & $\begin{array}{l}7 \\
5,00-8,00\end{array}$ & 2302,00 & $-2,12$ & $\begin{array}{l}p=.034 \\
p=.124\end{array}$ \\
\hline restrictions in sleeping & $\begin{array}{l}3 \\
1,00-6,00 \\
\end{array}$ & $\begin{array}{l}5 \\
3,00-7,00 \\
\end{array}$ & 1789,00 & $-3,91$ & $\begin{array}{l}p<.001 \\
p<.001\end{array}$ \\
\hline $\begin{array}{l}\text { satisfaction with pain } \\
\text { management }\end{array}$ & $\begin{array}{l}8,00 \\
7,00-10,00\end{array}$ & $\begin{array}{l}7,00 \\
4,00-8,00\end{array}$ & 1960,50 & 3,50 & $\begin{array}{l}p<.001 \\
p<.001\end{array}$ \\
\hline
\end{tabular}

\begin{tabular}{|c|c|c|c|c|c|}
\hline & with morphine ${ }^{1}$ & Without morphine $^{1}$ & u-value & $z$-value & $p$-value ${ }^{2}$ \\
\hline nausea & $\begin{array}{l}1 \\
0,00-7,00\end{array}$ & $\begin{array}{l}0 \\
0,00-1,50 \\
\end{array}$ & 2089,00 & $-3,16$ & $\begin{array}{l}p=.002 \\
p=.012\end{array}$ \\
\hline dizziness & $\begin{array}{l}3 \\
0,00-6,00\end{array}$ & $\begin{array}{l}1 \\
0,00-3,00\end{array}$ & 2078,00 & $-2,89$ & $\begin{array}{l}p=.004 \\
p=.020\end{array}$ \\
\hline obstipation & $\begin{array}{l}0 \\
0,00-3,00 \\
\end{array}$ & $\begin{array}{l}0 \\
0,00-4,00 \\
\end{array}$ & 329,50 & $-0,36$ & $\begin{array}{l}p=.717 \\
p=1.00\end{array}$ \\
\hline pruritus & $\begin{array}{l}3 \\
0,00-7,00\end{array}$ & $\begin{array}{l}2 \\
0,00-4,00\end{array}$ & 2173,00 & $-2,63$ & $\begin{array}{l}p=.009 \\
p=.036\end{array}$ \\
\hline back pain & $\begin{array}{l}1,50 \\
0,00-4,25\end{array}$ & $\begin{array}{l}1 \\
0,00-4,00\end{array}$ & 451,50 & $-0,20$ & $\begin{array}{l}p=.839 \\
p=1.00\end{array}$ \\
\hline headaches & $\begin{array}{l}0 \\
0,00-0,00\end{array}$ & $\begin{array}{l}0 \\
0,00-1,00\end{array}$ & 2078,00 & $-2,89$ & $\begin{array}{l}p=327 \\
p=.981\end{array}$ \\
\hline
\end{tabular}

\begin{tabular}{|l|l|l|l|l|l|}
\hline & ${\text { with } \text { morphine }^{1}}^{1}$ & Without morphine $^{1}$ & u-value & z-value & $\mathrm{p}$-value ${ }^{2}$ \\
\hline paracetamol (mg) & $0(32)$ & $1000(43)$ & 2185 & $-2,91$ & $\mathrm{p}=.004$ \\
& $0,00-1000,00$ & $0,00-1000,00$ & & & $\mathrm{p}=.020$ \\
\hline piritramid (mg) & $0(38)$ & $2(36)$ & 2834,50 & $-0,28$ & $\mathrm{p}=.781$ \\
& $0,00-5,00$ & $0,00-5,00$ & & & $\mathrm{p}=1.00$ \\
\hline ibuprofen (mg) & $800(53)$ & $800(55)$ & 2524 & $-1,43$ & $\mathrm{p}=.152$ \\
& $0,00-1600,00$ & $600,00-1600,00$ & & & $\mathrm{p}=.456$ \\
\hline diclofenac (mg) & $100(43)$ & $100(46)$ & 2368 & $-2,11$ & $\mathrm{p}=.035$ \\
& $0,00-100,00$ & $0,00-125,00$ & & & $\mathrm{p}=.140$ \\
\hline tramadol (mg) & $0(6)$ & $0(17)$ & 2383,5 & $-3,07$ & $\mathrm{p}=.002$ \\
& $0,00-0,00$ & $0,00-9,38$ & & & $\mathrm{p}=.012$ \\
\hline
\end{tabular}

Inclusion criteria: elective caesarean section ( $\geq 37$ th week of pregnancy), age $>18$ years, ASA I-III, and informed consent for regional anaesthesia 